\title{
Stem cells display a donor dependent response to escalating levels of growth factor release from extracellular matrix-derived scaffolds
}

Henrique V. Almeida ${ }^{\text {a, b }}$, Kevin J. Mulhall ${ }^{c}$, Fergal J. O’Brien ${ }^{\text {a, d, e, }}$, Daniel J.

$$
\text { Kelly }{ }^{\text {a, b, d, e * }}
$$

${ }^{a}$ Trinity Centre for Bioengineering, Trinity Biomedical Sciences Institute, Trinity College Dublin, Dublin 2, Ireland

b Department of Mechanical and Manufacturing Engineering, School of Engineering, Trinity College Dublin, Dublin 2, Ireland

${ }^{c}$ Sports Surgery Clinic, Santry, Dublin 9, Ireland

${ }^{d}$ Advanced Materials and Bioengineering Research Centre (AMBER), Trinity College Dublin \& Royal College of Surgeons in Ireland, Dublin 2, Ireland

e Tissue Engineering Research Group, Department of Anatomy, Royal College of Surgeons in Ireland, Dublin 2, Ireland

${ }^{*}$ Corresponding author: Daniel J. Kelly, Ph.D.

Address: Department of Mechanical and Manufacturing Engineering, School of Engineering, Parson's Building Trinity College Dublin, Dublin 2, Ireland Telephone: +353-1-896-3947, Fax: +353-1-679-5554

E-mail address: kellyd9@tcd.ie 
Abstract: Numerous growth factor delivery systems have been developed for tissue engineering. However, little is known about how the dose of a specific protein will influence tissue regeneration, or how different patients will respond to altered levels of growth factor presentation. The objective of this study was to assess stem cell chondrogenesis within extracellular-matrix (ECM)-derived scaffolds loaded with escalating levels of TGF- $\beta 3$. It was also sought to determine if stem cells display a donor dependent response to different doses of TGF- $\beta 3$ (low-5 ng to high-200 ng) released from such scaffolds. It was found that ECM-derived scaffolds possess the capacity to bind and release increasing amounts of TGF- $\beta 3$, with between $60-75 \%$ of this growth factor released into the media over the first 12 days of culture. After seeding these scaffolds with human infrapatellar fat pad-derived stem cells (FPSCs), it was found that cartilage specific ECM accumulation was greatest for the higher levels of growth factor loading. Importantly, soak-loading cartilage ECM-derived scaffolds with high levels of TGF- $\beta 3$ always resulted in at least comparable levels of chondrogenesis as continuously supplementing the media with this growth factor. Similar results were observed for FPSCs from all donors, although the absolute level of secreted matrix did vary from donor-to-donor. Therefore while no single growth factor release profile will be optimal for all patients, the results of this study suggest that the combination of a highly porous cartilage ECMderived scaffold, coupled with appropriate levels of TGF- $\beta 3$, can consistently drive chondrogenesis of adult stem cells.

Keywords: Cartilage; Extracellular matrix (ECM); Stem cells; TGF- $\beta$; Donor variability; Chondrogenesis; Growth factor dosage. 


\section{Introduction}

Articular cartilage regeneration still is a challenge in orthopaedic medicine. While cell based therapies have been reasonably successful clinically (Hunziker, 2002; Madeira et al., 2015), they are expensive and do not consistently result in hyaline cartilage regeneration. Tissue repair can potentially be augmented by the use of porous scaffolds to provide an environment more conductive to regeneration (O'Brien, 2011; Vinatier et al., 2009). Extracellular matrix (ECM)-derived scaffolds have shown particular promise in this regard, having been used in the repair of different tissues including cartilage (Almeida et al., 2015; Almeida et al., 2014; Benders et al., 2013; Cheng et al., 2014; Cheng et al., 2009; Cheng et al., 2011; Cheng et al., 2013; Diekman et al., 2010; Rowland et al., 2013; Sutherland et al., 2015).

Chondrogenesis is enhanced in the presence of members of the transforming growth factor (TGF)- $\beta$ family of growth factors, motivating the delivery of such proteins to articular cartilage defects to enhance regeneration (Madry et al., 2014). This multifunctional cytokine not only modulates stem cell differentiation, but has also been shown to regulate chondrocyte proliferation and matrix turnover in articular cartilage (Albro et al., 2013; Roberts et al., 1990). Various scaffolds and hydrogels can be used to deliver cells and growth factors such as TGF- $\beta$, thereby opening up the possibility of using such functionalized biomaterials to enhance chondrogenesis during articular cartilage repair (Almeida et al., 2015; Almeida et al., 2014; Benders et al., 2013; Cheng et al., 2014). However in the context of TGF- $\beta$ mediated articular cartilage regeneration, the optimal growth factor dose or release profile is still unclear 
(Madry et al., 2014; Tang et al., 2009). Furthermore, it remains unclear how different patients will respond to altered levels of growth factor delivery.

A number of different strategies have been developed to control the release of growth factors to accelerate tissue repair (Madry et al., 2014). TGF- $\beta$ is widely used to promote chondrogenesis in vitro (Madry et al., 2014; Tang et al., 2009), however its short half-life in vivo can limit its availability following delivery (Dinbergs et al.,1996; Madry et al., 2014; Nimni, 1997). Therefore in the context of articular cartilage regeneration, it is crucial to develop scaffolds to temporally and spatially control the release of TGF- $\beta$ to direct the differentiation of stem cells down a chondrogenic pathway (Madry et al., 2014; Tang et al., 2009). Established in vitro culture protocols that have been optimized to drive chondrogenesis can provide clues as to the optimal TGF- $\beta$ dosing and release rates required to drive chondrogenesis using growth factor delivery systems. Specifically, temporal exposure (2 weeks) to specific concentrations $(10 \mathrm{ng} / \mathrm{ml})$ of TGF- $\beta$ has been shown to promote robust chondrogenesis in vitro (Chung and Burdick, 2009; Fan et al., 2008; Shintani and Hunziker, 2011). Furthermore, it is known that continuous stimulation with TGF- $\beta$ can promote undesirable pathologies such as fibrosis and osteophyte formation (Blaney et al., 2006; Border and Ruoslahti, 1992; Elford et al., 1992; Kopesky et al., 2011; Madry et al., 2014; Mi et al., 2003; Mierisch et al., 2002; Nimni, 1997; van den Berg et al., 1993; van der Kraan and van den Berg, 2007; Wilson et al., 2009). This motivates the development of delivery systems capable of supplying growth factor within a 2 week dosing window (Bian et al., 2011; Mehlhorn et al., 2006). Different approaches have been used for exogenous chondrogenic 
growth factor delivery for cartilage tissue engineering, including from scaffolds (Almeida et al., 2015; Almeida et al., 2014; Matsiko et al., 2015) and/or from microsphere delivery systems (Ahearne et al., 2011; Dikina et al., 2015; Solorio et al., 2012; Solorio et al., 2012). Delivery of TGF- $\beta$ from cartilage ECM-derived scaffolds is particularly promising in this regard, as the biomaterial itself is inherently chondrogenic, and furthermore, this growth factor is known to bind strongly to ECM (Dinbergs et al., 1996). The negatively charged proteoglycans present in the pericellular matrix and ECM have been shown to bind and modulate the positively charged TGF- $\beta 3$, thereby regulating the availability of such growth factors (Macri et al., 2007).

Hence, the overall objective of this study was to assess the effect of delivering different doses of TGF- $\beta 3$ from cartilage ECM-derived scaffolds on chondrogenesis of human infrapatellar fat pad-derived stem cells (FPSCs). Furthermore, given the well documented donor-to-donor variability that exists in stem cell populations (Koller et al., 1996; Phinney et al., 1999), it was also sought to determine the effects of delivering different doses of TGF- $\beta 3$ from ECM-derived scaffold on FPSCs isolated from a range of healthy and diseased (osteoarthritic) donors.

\section{Material and methods}

\subsection{ECM-derived scaffold preparation}

The scaffolds were fabricated using a protocol previously developed (Almeida et al., 2015). Briefly, cartilage used in the fabrication of ECM-derived scaffolds was harvested under sterile conditions from the femoral condyles of three months old female pigs. Cartilage was first broken-up into small pieces 
using a scalpel. Furthermore, the scaffolds were fabricated using pulverized cartilage processed by a cryogenic mill (6770 Freezer/Mill, SPEX, UK). Then the slurry was homogenized in ultra pure water (UPW) using a homogenizer (IKAT10, IKA Works Inc, NC, USA) to create a fine cartilage slurry of $250 \mathrm{mg}$ of particulated cartilage for $1 \mathrm{ml}$ of UPW. The slurry was transferred to custom made moulds (containing wells $5 \mathrm{~mm}$ in diameter and $3 \mathrm{~mm}$ in height) and freeze-dried (FreeZone Triad, Labconco, KC, USA) to produce porous scaffolds, as previously described (Almeida et al., 2015). Briefly, the slurry was frozen to $-30^{\circ} \mathrm{C}\left(1^{\circ} \mathrm{C} / \mathrm{min}\right)$ and kept at that temperature for one hour. Followed by an increase to $-10^{\circ} \mathrm{C}\left(1^{\circ} \mathrm{C} / \mathrm{min}\right)$ under vacuum for 24 hours and then finally to room temperature $\left(0.5^{\circ} \mathrm{C} / \mathrm{min}\right)$. Subsequently, two different crosslinking techniques were applied to the scaffolds. The scaffolds underwent DHT and 1Ethyl-3-3dimethyl aminopropyl carbodiimide (EDAC) crosslinking as previously described in literature (Haugh et al., 2011). The DHT process was performed in a vacuum oven (VD23, Binder, Germany), at $115^{\circ} \mathrm{C}$, in 2 mbar for 24 hours. The EDAC (Sigma-Aldrich, Germany) crosslinking consisted of chemical exposure for 2 hours at a concentration of $6 \mathrm{mM}$ in the presence of $\mathrm{N}$-Hydroxysuccinimide (NHS) (Sigma-Aldrich, Germany), a catalyst that is commonly used with EDAC. A molar ratio of 2.5 M EDAC/M N-Hydroxysuccinimide was used (Haugh et al., 2011; Olde Damink et al., 1996).

\subsection{Scanning Electron Microscopy (SEM) and Light Microscopy}

Acellular ECM-derived scaffolds were imaged using scanning electron microscopy (SEM) (Figure 1). Structures were fixed in 4\% paraformaldehyde solution (PFA) overnight. Furthermore, scaffolds were dehydrated through 
successive graded ethanol baths (10-100\%), fixed onto aluminium stubs, coated with gold and examined under a field emission scanning electron microscope (Tescan Mira FEG-SEM XMU, Libušina, Czech Republic). Routine light microscopy was also used for morphometrical and histological analysis.

\subsection{Cell and construct culture}

Ethical approval for the isolation of human infrapatellar fat pad (IFP) derived stem cells (FPSCs) was obtained from the institutional review board of the Mater Misericordiae University Hospital Dublin. Cells were isolated from the IFP of patients undergoing total joint arthroplasty (diseased) or anterior cruciate ligament $(A C L)$ surgery (healthy). Cells (Donor A: Male, 26; Donor B: Male, 26; Donor C: Male, 19; Donor D: Male, 34; Donor E: Female, 67; Donor F: Female, 66) were isolated as previously described (Almeida et al., 2015). Briefly, IFPs were harvested, weighed and washed thoroughly in phosphate buffered saline (PBS) (Sigma-Aldrich, Germany). Next, the tissue was diced in sterile conditions and followed by rotation at $37^{\circ} \mathrm{C}$ in high-glucose Dulbecco's Modified Eagle Medium (hgDMEM, GlutaMAXTM)(GIBCO, Biosciences, Ireland) containing collagenase type II (750 U/ml, Worthington Biochemical, LaganBach Services, Ireland) and $1 \%$ penicillin $(100 \mathrm{U} / \mathrm{ml})$-streptomycin $(100 \mu \mathrm{g} / \mathrm{ml})$ for approximately 4 hours. A ratio of $4 \mathrm{ml}$ of collagenase $(750 \mathrm{U} / \mathrm{ml})$ per gram of tissue was found to be optimal based on previous work (Buckley and Kelly, 2012; Buckley et al., 2010; Liu et al., 2012). After digestion, cells were washed, filtered (40 $\mu \mathrm{m}$ nylon cell strainer) and centrifuged at $650 \mathrm{~g}$ for 5 minutes. The supernatant was removed. The remaining cells were re-suspended, counted and finally plated $\left(5 \times 10^{3}\right.$ cells $\left./ \mathrm{cm}^{2}\right)$ in T-175 flasks (Sarstedt, Wexford, Ireland). 
Cells were cultured in a standard media formulation, which consisted of hgDMEM containing $10 \%$ foetal bovine serum and $1 \%$ penicillin $(100 \mathrm{U} / \mathrm{ml})$ streptomycin $(100 \mathrm{mg} / \mathrm{ml})$ (GIBCO, Biosciences, Ireland) with the addition of fibroblast-growth factor-2 (FGF-2, $5 \mathrm{ng} / \mathrm{ml}$; ProSpec-Tany TechnoGene Ltd, Israel). Cells were expanded to passage 2 (P2), with an initial seeding density of $5 \times 10^{3}$ cells $/ \mathrm{cm}^{2}$ at each passage. Media changes were performed twice a week.

Each scaffold was seeded with $0.5 \times 10^{6}$ human FPSCs in $40 \mu \mathrm{l}$ of media. Constructs were maintained in chemically defined chondrogenic medium (CDM), as previously described, for 28 days $\left(\right.$ at $5 \% \mathrm{O}_{2}$ and $37^{\circ} \mathrm{C}$ ) (Almeida et al., 2015; Buckley and Kelly, 2012). CDM consisted of DMEM GlutaMAX ${ }^{\mathrm{TM}}$ supplemented with penicillin $(100 \mathrm{U} / \mathrm{ml})$-streptomycin $(100 \mu \mathrm{g} / \mathrm{ml})($ both GIBCO, Biosciences, Ireland), $100 \mu \mathrm{g} / \mathrm{ml}$ sodium pyruvate, $40 \mu \mathrm{g} / \mathrm{ml} \mathrm{L-proline,} 50 \mu \mathrm{g} / \mathrm{ml}$ L-ascorbic acid-2-phosphate, $1.5 \mathrm{mg} / \mathrm{ml}$ BSA, 1x insulin-transferrin-selenium, $100 \mathrm{nM}$ dexamethasone (all from Sigma-Aldrich, Ireland) and $10 \mathrm{ng} / \mathrm{ml}$ recombinant human growth factor- $\beta 3$ (TGF- $\beta 3$; ProSpec-Tany TechnoGene Ltd, Israel). For groups described in the text as loaded with TGF- $\beta 3$, additional TGF$\beta 3$ was not added to the media during the culture period. Instead, TGF- $\beta 3$ was soak loaded into the scaffold and was not directly added to the culture media. For the first part of the study, where the dosing effects were first assessed for a single donor, the experimental groups were: Low dose (3 ng), Medium (30 ng), High (300 ng) and Media supplemented (10 ng/ml). For the second part of this study, six different cell donors were assessed with low (5 ng), medium (50 ng), high (200 ng) and media supplementation $(10 \mathrm{ng} / \mathrm{ml})$ growth factor dosage. 
(Note: For a typical chondrogenic culture media - $10 \mathrm{ng} / \mathrm{ml}$ of TGF- $\beta 3,2.5 \mathrm{ml}$ of media per scaffold, 2 media changes per week - the total amount of TGF- $\beta 3$ used per week is $50 \mathrm{ng}$. This equates to $200-300 \mathrm{ng}$ of growth factor over a 4-6 week culture period. This motivated the choice of the dosing for the 'high' TGF$\beta 3$ groups). For all experiments, the scaffolds were kept in 12 well plates (1 scaffold per well) and each scaffold was placed within cylindrical agarose moulds. After cell seeding, the scaffolds were kept in the incubator for two hours. After two hours, $2.5 \mathrm{ml}$ of media was added to each well. Media changes were performed twice a week. The media was stored at $-85^{\circ} \mathrm{C}$ for further analysis.

\subsection{Biochemical analysis}

Constructs were biochemically analyzed at day 28, for sulphated glycosaminoglycan (sGAG) and collagen content $(n=6)$, as previously described (Almeida et al., 2015; Buckley et al., 2010). Briefly, constructs were enzymatically digested by incubation in papain $(125 \mu \mathrm{g} / \mathrm{ml})$ in $0.1 \mathrm{M}$ sodium acetate, $5 \mathrm{mM}$ cysteine $\mathrm{HCl}, 0.05 \mathrm{M}$ EDTA, pH 6.0 (all from Sigma-Aldrich, Ireland) at $60^{\circ} \mathrm{C}$ under rotation (10 rpm) for 18 hours. The proteoglycan content was estimated by quantifying the sGAG in constructs using the

dimethylmethylene blue dye-binding assay (Blyscan, Biocolor Ltd., Northern Ireland), using bovine chondroitin sulphate as a standard. Collagen content was determined by measuring hydroxyproline content, after acidic hydrolysis of the samples at $110^{\circ} \mathrm{C}$ for $18 \mathrm{~h}$ in concentrated $\mathrm{HCL}(38 \%)$. Samples were assayed using a chloramine-T assay assuming a hydroxyproline/collagen ratio of 1:7.69 (lgnat'eva et al., 2007). 


\subsection{Histology and immunohistochemistry}

Constructs were fixed overnight at $4^{\circ} \mathrm{C}$ in a $4 \%$ solution of paraformaldehyde (Sigma-Aldrich, Ireland), followed by washing in PBS (Sigma-Aldrich, Ireland), dehydrated and wax embedded, as previously described (Almeida et al., 2015). Wax embedded constructs were sectioned in 6 $\mu \mathrm{m}$ thick slices and mounted in microscope slides. Sections were stained with $1 \%$ Alcian blue $8 \mathrm{GX}$ (Sigma-Aldrich) in $0.1 \mathrm{M} \mathrm{HCl}$ for sGAG and with Picrosirius red for collagen. With the aim of monitoring the newly formed sGAG, constructs were histologically analysed (Alcian blue) at day 28. Immunohistochemical analysis was performed on $6 \mu \mathrm{m}$ sections using monoclonal antibody to type II collagen (Abcam, UK). Samples were washed in PBS and subjected to peroxidase activity (20 min), incubated $\left(1\right.$ hour, $37^{\circ} \mathrm{C}$ in a moist environment) with chondroitinase $\mathrm{ABC}$ (Sigma, $0.25 \mathrm{U} / \mathrm{ml}$ ) with the aim of enhancing the permeability of the ECM by removing the chondroitin sulphate. Slides were rinsed with PBS and blocked with 10\% goat serum (30 minutes) and incubated with mouse monoclonal anti-collagen type II diluted 1:100 (Abcam, UK; concentration $1 \mathrm{mg} / \mathrm{ml} ; 1$ hour at RT). A secondary antibody for type II collagen (Anti-Mouse IgG Biotin antibody produced in goat; concentration $1 \mathrm{~g} / \mathrm{L}$ ) binding was then applied (1 hour). By using Vectastain ABC reagent (Vectastain ABC kit, Vector Laboratories, UK) for $5 \mathrm{~min}$ in peroxidase DAB substrate kit (Vector laboratories, UK), it was possible to observe a colour alteration. Samples were dehydrated with graded ethanol and xylene and mounted with Vectamount medium (Vector Laboratories, UK). 


\subsection{Measurement of TGF- $\beta 3$ release from ECM scaffolds}

TGF- $\beta 3$ release from acellular scaffolds was determined via ELISA, as previously described (Albro et al., 2013; Almeida et al., 2014; Vonwil et al., 2008). 96 well plates were coated with capture antibody $(360 \mu \mathrm{g} / \mathrm{ml})$ with mouse anti-human TGF- $\beta 3$ (R\&D Systems, UK). Samples and TGF- $\beta 3$ standards (ProSpec-Tany TechnoGene Ltd, Israel) were incubated for 2 hours. After washing and drying, detection antibody $(18 \mu \mathrm{g} / \mathrm{ml}$ of biotinylated goat antihuman TGF- $\beta 3$ ) was added to the plate and incubated (2 hours). The next step was to wash, dry and incubate the plate in streptavidin-HRP (horseradishperoxidase; R\&D Systems, UK) for 20 minutes in the dark. Substrate solution (1:1 mixture of $\mathrm{H}_{2} \mathrm{O}_{2}$ and tetramethylbenzidine; R\&D Systems, UK) was added to each well, followed by incubation (20 minutes) avoiding direct light. Stop solution (2 $\mathrm{N} \mathrm{H}_{2} \mathrm{SO}_{4}$; Sigma-Aldrich, Germany) was added and the optical density was determined immediately with a plate reader $(450 \mathrm{~nm})$.

\subsection{Statistical analysis}

Results are presented as mean \pm standard deviation. Statistical analysis was performed with MINITAB 15.1 software package (Minitab Ltd., Coventry, UK). Experimental groups were analyzed for significant differences using a general linear model for analysis of variance with factors including growth factor dose and cell donors. Tukey's test for multiple comparisons was used to compare conditions. Significance was accepted at a level of $p<0.05$. 


\section{Results}

\subsection{Cartilage ECM-derived scaffolds can bind and control the release of escalating doses of TGF- $\beta 3$}

Three different doses (low-3 ng, medium-30 ng and high-300ng) of TGF$\beta 3$ were loaded onto cartilage ECM derived scaffolds, which were then seeded with human FPSCs and maintained in vitro for 4 weeks. Cartilage ECM-derived scaffolds possessed a consistent capacity to bind and release increasing amounts of TGF- $\beta 3$ (Figure 2). Irrespective of the concentration of TGF- $\beta 3$ applied, the scaffolds released between $60-75 \%$ of the loaded growth factor into the media over the first 12 days of culture. Over this time period, the low group released a total of $2.4 \pm 0.2 \mathrm{ng}$ of TGF- $\beta 3$ (Figure $2 \mathrm{~A}$ ), the medium group released 21.4 $\pm 2.3 \mathrm{ng}$ (Figure 2B) and high dose group released 198.0 $\pm 25.4 \mathrm{ng}$ (Figure 2C). In terms of the total percentage of growth factor released over 12 days, this corresponded to $74.0 \pm 6.0 \%$ of loaded TGF- $\beta 3$ released from the low group (Figure 2D), $67.0 \pm 7.0 \%$ from the medium group (Figure $2 \mathrm{E}$ ) and finally $60.8 \pm 7.0 \%$ from the high group (Figure $2 \mathrm{~F}$ ).

\subsection{Chondrogenesis within ECM-derived scaffolds seeded with human stem cells strongly depends on the dose of TGF- $\beta 3$ loaded into the construct}

After 4 weeks in culture, only scaffolds loaded with either medium or high doses of TGF- $\beta 3$ resembled cartilage macroscopically (Figure 3 I and M). These engineered tissues also appeared macroscopically similar to constructs were TGF- $\beta 3$ was continuously added to the culture media (Figure 3Q; Note: a total 
of $200 \mathrm{ng}$ of TGF- $\beta 3$ was added to the media of this group over the 28 day culture period). Histologically, very little cartilage matrix deposition was observed in scaffolds that were not exogenously stimulated with TGF- $\beta 3$ (Figure 3B-D), appearing similar to day-zero scaffolds (data not shown). Alcian Blue staining suggested little sGAG accumulation within scaffolds loaded with low and medium doses of TGF- $\beta 3$ (Figure $3 \mathrm{~F}$ and J). Conversely, robust sGAG deposition was observed in scaffolds loaded with high levels of TGF- $\beta 3$, or where the growth factor was directly supplemented in the culture media (Figure $3 \mathrm{~N}$ and R). Collagen matrix staining was strong for medium dose (Figure $3 \mathrm{~K}$ ), high dose (Figure 30) and media supplemented (Figure 3S) scaffolds. Staining for type II collagen was most intense within scaffolds loaded with high doses of TGF- $\beta 3$ (Figure 3P).

Total sGAG content after 4 weeks of culture period was highest in scaffolds loaded with a high dose of TGF- $\beta 3$, being at least comparable to constructs where the growth factor was continuously supplemented to the media (Figure 4A). Collagen content was significantly lower for the low dose group when compared with the other scaffolds (Figure 4B).

\subsection{Stem cells display a donor dependent response to TGF- $\beta 3$ delivery from ECM-derived scaffolds}

Having demonstrated that cartilage ECM derived scaffolds can be used to control the delivery of different doses of TGF- $\beta 3$, and that chondrogenesis within these scaffolds was dependent on the amount of growth factor loaded onto the scaffold, we next sought to determine if FPSCs display a donor dependant response to the release of different doses of TGF- $\beta 3$ from these 
constructs. To this end, FPSCs from a range of healthy and diseased donors were used. After 4 weeks in culture, sGAG staining (alcian blue) was weak for all six donors $(A-F)(E$ and $F$ osteoarthritic - OA) when a low dose (5 ng per scaffold) of TGF- $\beta 3$ was loaded onto the scaffolds (Figure 5). For a medium dose (50 ng) of growth factor, reasonable levels of chondrogenesis (as evidenced by Alcian Blue staining for sGAG deposition) were observed for 2 of the 6 donors (donor $\mathrm{C}$ and $\mathrm{F}$ ). With the exception of donor $\mathrm{A}$, robust chondrogenesis was observed in all cases when high doses of TGF- $\beta 3$ were loaded onto the scaffolds (Figure 5). Robust collagen deposition (picro-sirius red staining) was observed for all donors in all conditions, with the exception of donor A when stimulated with a low dose of growth factor (Figure 5). In agreement with previous studies using a pellet culture system (Liu et al., 2014), the disease state of the donors (either healthy or osteoarthritic) did not appear to impact chondrogenesis of FPSCs (Figure 5).

Quantification of the total SGAG content after 28 days of culture within constructs initially loaded with increasing doses of TGF- $\beta 3$ generally agreed with the histological analysis of the engineered tissues (Figure 6). For all donors, the highest levels of sGAG accumulation were observed in scaffolds loaded with high doses of TGF- $\beta 3$. For this high dose, sGAG accumulation was highest for donor $\mathrm{B}$, reaching close to $470 \mu \mathrm{g}$, in comparison to approximately $260 \mu \mathrm{g}$ for donor A. Again, there was no evidence of diminished sGAG deposition using FPSCs isolated from diseased (OA) donors (Figure 6). 


\section{Discussion}

The overall goal of this study was to assess the capacity of cartilage ECM-derived scaffolds to bind and release different amounts of soak loaded TGF- $\beta 3$. Furthermore, it was also sought to determine if adult stem cells display a donor dependent response to the binding and release of different doses of TGF- $\beta 3$ from such ECM-derived scaffolds. These scaffolds possessed a significant capacity to bind and deliver increasing amounts of TGF- $\beta 3$. They released between $60-75 \%$ of the loaded TGF- $\beta 3$ into the media over 12 days of culture, irrespective of the concentration of growth factor added to the scaffold, with the remainder apparently sequestered within the ECM. After a 28 day culture period, only constructs loaded with either medium or high doses of TGFß3 resembled cartilage macroscopically. Histologically, very little cartilage matrix deposition was observed in scaffolds that were not stimulated with TGFß3. Robust sGAG and collagen deposition was observed in scaffolds loaded with high levels of growth factor, or where the TGF- $\beta 3$ was regularly supplemented into the media. It was also observed that staining for type II collagen, the main collagen type in articular cartilage, was most intense within scaffolds loaded with high doses of TGF- $\beta 3$. Having demonstrated that cartilage ECM-derived scaffolds can be used to control the delivery of different doses of TGF- $\beta 3$, and that chondrogenesis within these scaffolds was dependent on the amount of growth factor loaded onto the scaffold, we next sought to determine if FPSCs display a donor dependant response to the release of different doses of TGF- $\beta 3$ from these constructs. To this end, FPSCs from a range of healthy and diseased donors were seeded into cartilage ECM derived scaffolds. 
Chondrogenesis was observed in most of the cases when high doses of growth factor were used. The donor's disease state (healthy or osteoarthritic) did not impact chondrogenesis in these ECM derived scaffolds.

The release profile of TGF- $\beta 3$ from cartilage ECM-derived scaffolds was relatively insensitive to the dose of growth factor loaded into the stem cell laden construct, with between 60 and $75 \%$ of the growth factor released over the first 12 days of culture. The growth factor interaction with the scaffold is possible due to known non-specific binding sites present in native cartilage ECM (Albro et al., 2013). Binding sites for TGF- $\beta$ include proteoglycans, glycosaminoglycans, collagens and glycoproteins, and these interactions are known to influence growth factor availability (Albro et al., 2013). Previous studies have used gelatin (denatured collagen) microspheres to control the release of growth factors such as TGF- $\beta$ (Ahearne et al., 2011; Holland et al., 2003). Positively charged growth factors can be absorbed by these lyophilized microspheres by creation of a polyion complex with gelatin (Ahearne et al., 2011; Holland et al., 2003). TGF- $\beta$ is released when this complex is compromised, or via material degradation due to cell mediated proteolysis. Additionally, the rate of release of such growth factor is dependent on the polymer degree of crosslinking (Ahearne et al., 2011; Young et al., 2005). With these gelatin microspheres, approximately $10 \%$ of loaded TGF- $\beta 1$ is released over 15 days. Chondroitin/hyaluronic-acid scaffolds containing gelatin microspheres have also been used as TGF- $\beta 3$ delivery systems, which showed an initial burst release of $37 \%$ of loaded growth factor, with $80 \%$ released after 18 days (Fan et al., 2006a; Fan et al., 2006b). Other examples include a hybrid 
system consisting of type I/II collagen and TGF- $\beta 1$-loaded fibrin (Dickhut et al., 2010), and a heparin/fibrin/PCL system which supported in situ chondrogenesis of adipose-derived stem cells while releasing approximately $65 \%$ of the initial TGF- $\beta 1$ loaded into the construct during the first week of culture (Jung et al., 2009). The release of bound growth factor from stem cell laden scaffolds may also depend on rate of new tissue development within the scaffold, which may act as a new sink for TGF- $\beta 3$ as it is released from the scaffold. Higher levels of new tissue is deposited within scaffolds loaded with the high doses of exogenous TGF- $\beta 3$, which may explain why a higher total amount of growth factor appears sequestered within these construct after 12 days of culture.

In agreement with previous studies (Almeida et al., 2015), exogenous growth factor (TGF- $\beta 3$ ) stimulation was necessary to induce chondrogenesis of FPSCs, even in the presence of native cartilage ECM. Cartilage specific matrix accumulation within these FPSCs seeded scaffolds was greatest for the higher levels of growth factor loading. It has previously been shown that TGF- $\beta$ enhances chondrogenesis of stem cells in a dose dependent manner (Worster et al., 2000). The gold standard for promoting chondrogenesis of mesenchymal stem cells in vitro is to supplement the media with $10 \mathrm{ng} / \mathrm{ml}$ of TGF- $\beta 3$; however the dosage required to drive chondrogenesis in vivo from a growth factor delivery scaffold has yet to be elucidated. Previously reported TGF- $\beta$ dosing studies have demonstrated that a brief exposure to high concentrations of TGF$\beta(100 \mathrm{ng} / \mathrm{ml}$ for $30 \mathrm{~min}$ ) was beneficial to chondrogenesis (Miura et al., 2002). This suggests that the TGF- $\beta$ burst release from the ECM-derived scaffold observed in the first three days of the current study may be advantageous for 
driving robust chondrogenesis of stem cells seeded in the scaffold. There are, however, potential drawbacks associated with excessive TGF- $\beta$ release from a scaffold into the joint space that need to be considered when developing any growth factor delivery system. For example, it has previously been reported that 21 days after a single joint injection of 200 ng of TGF- $\beta 1$, sGAG synthesis was significantly increased in a murine knee joint model (van Beuningen et al., 1998). However, repeated growth factor injections can lead to TGF- $\beta 1$-induced osteophyte formation (van Beuningen et al., 1998). Therefore, there is a clear need to perform additional in vivo studies to identify the dose of growth factor which will accelerate articular cartilage regeneration without promoting undesirable side effects.

Human stem cells displayed a donor dependant response to escalating levels of exogenous growth factor stimulation within ECM-derived scaffolds, although importantly the greatest levels of chondrogenesis were consistently observed within constructs loaded with the higher levels of TGF- $\beta 3$. It is known that bone marrow-derived mesenchymal stem cells from different donors may have dissimilar responses to environmental stimuli (Koller et al., 1996; Phinney et al., 1999; Pilgaard et al., 2009; Solorio et al., 2012). In the current study we observed that if the dose of TGF- $\beta 3$ loaded onto cartilage ECM derive scaffolds is increased (specifically to the high dose), similar level of chondrogenesis by infrapatellar fat pad derived stem cells from a range of healthy and diseased donors was found. This suggests that the combination of an optimized chondroinductive scaffolds (Almeida et al., 2015), coupled with appropriate growth factor presentation, can minimize the donor-to-donor variability in 
chondrogenesis traditionally associated with adult human stem cells. Such consistency in outcome will facilitate the development, and future implementation, of more effective treatments for human cartilage repair.

\section{Conclusion}

This study demonstrates that by delivering a high dose of TGF- $\beta 3$ from a cartilage ECM-derived scaffold that it is possible to minimize donor-to-donor variability in chondrogenesis of human infrapatellar fat pad-derived stem cells. Furthermore, no major difference was observed in the chondrogenic capacity of FPSCs from healthy and diseased donors when seeded onto such growth factor delivery scaffolds. Such consistency in chondrogenesis across a range of human donors should facilitate the future clinical translation of such treatments for articular cartilage regeneration.

\section{Acknowledgments}

Funding for this study was provided by a European Research Council Starter Grant (StemRepair - Project number: 258463), Programme for Research in Third-Level Institutions (PRTLI) - Graduate Research Education Programme in Engineering, and co-funded by the European Regional Development Fund and the HEA. 


\section{References}

Ahearne M, Buckley CT, Kelly DJ. 2011, A growth factor delivery system for chondrogenic induction of infrapatellar fat pad-derived stem cells in fibrin hydrogels. Biotech and App Biochem, 58(5): 345-352

Albro MB, Nims RJ, Cigan AD, Yeroushalmi KJ, Alliston T, Hung CT, Ateshian GA. 2013, Accumulation of exogenous activated TGF- $\beta$ in the superficial zone of articular cartilage. Biophys J, 104(8): 1794-1804

Almeida HV, Cunniffe GM, Vinardell T, Buckley CT, O'Brien FJ, Kelly DJ. 2015, Coupling freshly isolated $\mathrm{CD} 44^{+}$infrapatellar fat pad-derived stromal cells with a TGF- $\beta 3$ eluting cartilage ECM-derived scaffold as a single-stage strategy for promoting chondrogenesis. Adv Healthc Mater, 4(7): 1043-53

Almeida HV, Liu Y, Cunniffe GM, Mulhall KJ, Matsiko A, Buckley CT, O'Brien FJ, Kelly DJ. 2014, Controlled release of transforming growth factor- $\beta 3$ from cartilage-extra-cellular-matrix-derived scaffolds to promote chondrogenesis of human-joint-tissue-derived stem cells. Acta Biomater, 10(10), 4400-4409

Benders KE, Weeren P, Badylak SF, Saris D, Dhert WA, Malda J. 2013, Extracellular matrix scaffolds for cartilage and bone regeneration, Trends in Biotechnology, 31(3): 169-176

Bian L, Zhai DY, Tous ER, Mauck RL, Burdick JA. 2011, Enhanced MSC chondrogenesis following delivery of TGF- $\beta 3$ from alginate microspheres within hyaluronic acid hydrogels in vitro and in vivo. Biomaterials, 32(27): $6425-6434$ 
Blaney Davidson EN, Vitters EL, van den Berg WB, van der Kraan PM. 2006, TGF $\beta$-induced cartilage repair is maintained but fibrosis is blocked in the presence of Smad7. Arthritis Res Ther, 8(3): R65

Border WA, Ruoslahti E. 1992, Transforming growth factor- $\beta$ in disease: the dark side of tissue repair. J Clin Invest, 90(1): 1-7

Buckley CT, Kelly DJ. 2012, Expansion in the presence of FGF-2 enhances the functional development of cartilaginous tissues engineered using infrapatellar fat pad derived MSCs. Journal of the Mech Behavior of Biomed Mat, 11: 102-111

Buckley CT, Vinardell T, Kelly DJ. 2010, Oxygen tension differentially regulates the functional properties of cartilaginous tissues engineered from infrapatellar fat pad derived MSCs and articular chondrocytes. Osteoarthritis and Cartilage, 18(10): 1345-1354

Buckley CT, Vinardell T, Thorpe SD, Haugh MG, Jones E, McGonagle D, Kelly DJ. 2010, Functional properties of cartilaginous tissues engineered from infrapatellar fat pad-derived mesenchymal stem cells. Journal of Biomechanics, 43(5): 920-926

Cheng CW, Solorio LD, Alsberg E. 2014, Decellularized tissue and cell-derived extracellular matrices as scaffolds for orthopaedic tissue engineering. Biotechnology Advances, 32(2): 462-484

Cheng NC, Estes BT, Awad HA, Guilak F. 2009, Chondrogenic differentiation of adipose-derived adult stem cells by a porous scaffold derived from native articular cartilage extracellular matrix. Tissue Eng Part A, 15(2): 231-241 
Cheng NC, Estes BT, Young TH, Guilak F. 2011, Engineered cartilage using primary chondrocytes cultured in a porous cartilage-derived matrix. Regen Med, 6(1): 81-93

Cheng NC, Estes BT, Young TH, Guilak F. 2013, Genipin-crosslinked cartilagederived matrix as a scaffold for human adipose-derived stem cell chondrogenesis. Tissue Eng Part A, 19(3-4): 484-96

Chung C, Burdick JA. 2009, Influence of three-dimensional hyaluronic acid microenvironments on mesenchymal stem cell chondrogenesis. Tissue Eng Part A, 15(2): 243-254

Dickhut A, Dexheimer V, Martin K, Lauinger R, Heisel C, Richter W. 2010, Chondrogenesis of human mesenchymal stem cells by local transforming growth factor- $\beta$ delivery in a biphasic resorbable carrier. Tissue Eng Part A, 16(2): 453-464

Diekman BO, Rowland CR, Lennon DP, Caplan Al, Guilak F. 2010, Chondrogenesis of adult stem cells from adipose tissue and bone marrow: induction by growth factors and cartilage-derived matrix. Tissue Eng Part A, 16(2): 523-533

Dikina AD, Strobel HA, Lai BP, Rolle MW, Alsberg E. 2015, Engineered cartilaginous tubes for tracheal tissue replacement via self-assembly and fusion of human mesenchymal stem cell constructs. Biomaterials, 52: $452-462$

Dinbergs ID, Brown L, Edelman ER. 1996, Cellular response to transforming growth factor- $\beta 1$ and basic fibroblast growth factor depends on release 
kinetics and extracellular matrix interactions. J Biol Chem, 271(47): $29822-29829$

Elford PR, Graeber M, Ohtsu H, Aeberhard M, Legendre B, Wishart WL, MacKenzie AR. 1992, Induction of swelling, synovial hyperplasia and cartilage proteoglycan loss upon intra-articular injection of transforming growth factor beta-2 in the rabbit. Cytokine, 4(3), 232-238

Fan H, Hu Y, Li X, Wu H, Lv R, Bai J, Wang J, Qin L. 2006 a, Ectopic cartilage formation induced by mesenchymal stem cells on porous gelatinchondroitin-hyaluronate scaffold containing microspheres loaded with TGF- $\beta 1$. Int J Artif Organs, 29(6): 602-611

Fan H, Hu Y, Qin L, Li X, Wu H, Lv R. 2006 b, Porous gelatin-chondroitinhyaluronate tri-copolymer scaffold containing microspheres loaded with TGF- $\beta 1$ induces differentiation of mesenchymal stem cells in vivo for enhancing cartilage repair. J Biomed Mater Res A, 77(4):785-794

Fan H, Zhang C, Li J, Bi L, Qin L, Wu H, Hu Y. 2008, Gelatin Microspheres Containing TGF- $\beta 3$ Enhance the Chondrogenesis of Mesenchymal Stem Cells in Modified Pellet Culture. Biomacromolecules, 9(3): 927-934

Haugh MG, Murphy CM, McKiernan RC, Altenbuchner C, O'Brien FJ. 2011, Crosslinking and mechanical properties significantly influence cell attachment, proliferation, and migration within collagen glycosaminoglycan scaffolds. Tissue Engineering Part A, 17(9-10): 12011208

Holland TA, Tabata Y, Mikos AG. 2003, In vitro release of transforming growth factor- $\beta 1$ from gelatin microparticles encapsulated in biodegradable, 
injectable oligo(poly(ethylene glycol) fumarate) hydrogels. Journal of Controlled Release, 91: 299-313.

Hunziker EB. 2002, Articular cartilage repair: basic science and clinical progress. A review of the current status and prospects. Osteoarthritis and Cartilage, 10(6): $432-463$

Ignat'eva NY, Danilov NA, Averkiev SV, Obrezkova MV, Lunin VV, Sobol EN. 2007, Determination of hydroxyproline in tissues and the evaluation of the collagen content of the tissues. Journal of Analytical Chemistry, 62(1): $51-57$

Jung Y, Chung YI, Kim SH, Tae G, Kim YH, Rhie JW, Kim SH. 2009, In situ chondrogenic differentiation of human adipose tissue-derived stem cells in a TGF- $\beta 1$ loaded fibrin-poly(lactide-caprolactone) nanoparticulate complex. Biomaterials, 30(27): 4657-4664

Koller MR, Manchel I, Brott DA, Palsson B. 1996, Donor-to-donor variability in the expansion potential of human bone marrow cells is reduced by accessory cells but not by soluble growth factors. Exp Hematol, 24(13): $1484-1493$

Kopesky PW, Vanderploeg EJ, Kisiday JD, Frisbie DD, Sandy JD, Grodzinsky AJ. 2011, Controlled delivery of transforming growth factor- $\beta 1$ by selfassembling peptide hydrogels induces chondrogenesis of bone marrow stromal cells and modulates smad2/3 signaling. Tissue Engineering Part A, 17(1-2): 83-92

Liu Y, Buckley CT, Almeida HV, Mulhall KJ, Kelly DJ. 2014, Infrapatellar fat pad-derived stem cells maintain their chondrogenic capacity in disease 
and can be used to engineer cartilaginous grafts of clinically relevant dimensions. Tissue Eng Part A, 20(21-22): 3050-3062

Liu Y, Buckley CT, Downey R, Mulhall KJ, Kelly DJ. 2012, The role of environmental factors in regulating the development of cartilaginous grafts engineered using osteoarthritic human infrapatellar fat pad-derived stem cells. Tissue Engineering Part A, 18(15-16): 1531-1541

Macri L, Silverstein D, Clark RF. 2007, Growth factor binding to the pericellular matrix and its importance in tissue engineering. Adv Drug Delivery Reviews, 59(13): 1366-1381

Madeira C, Santhagunam A, Salgueiro JB, Cabral JM. 2015, Advanced cell therapies for articular cartilage regeneration. Trends Biotechnol, 33(1): $35-42$

Madry H, Rey-Rico A, Venkatesan JK, Johnstone B, Cucchiarini M. 2014, Transforming growth factor $\beta$-releasing scaffolds for cartilage tissue engineering. Tissue Eng Part B Rev, 20(2): 106-125

Matsiko A, Levingstone TJ, Gleeson JP, O'Brien FJ. 2015, Incorporation of TGF- $\beta 3$ within collagen-hyaluronic acid scaffolds improves their chondrogenic potential. Adv Healthc Mater, 4(8): 1175-1179

Mehlhorn AT, Schmal H, Kaiser S, Lepski G, Finkenzeller G, Stark GB, Sudkamp NP. 2006, Mesenchymal stem cells maintain TGF- $\beta$-mediated chondrogenic phenotype in alginate bead culture. Tissue Eng, 12(6): 1393-1403

Mi Z, Ghivizzani SC, Lechman E, Glorioso JC, Evans CH, Robbins PD. 2003, Adverse effects of adenovirus-mediated gene transfer of human 
transforming growth factor- $\beta 1$ into rabbit knees. Arthritis Res Ther, 5(3): $1-8$

Mierisch CM, Cohen SB, Jordan LC, Robertson PG, Balian G, Diduch DR. 2002, Transforming growth factor- $\beta$ in calcium alginate beads for the treatment of articular cartilage defects in the rabbit. Arthroscopy: The Journal of Arthroscopic \& Related Surgery, 18(8): 892-900

Miura Y, Parvizi J, Fitzsimmons JS, O'Driscoll SW. 2002, Brief exposure to high-dose transforming growth factor- $\beta 1$ enhances periosteal chondrogenesis in vitro: a preliminary report. J Bone Joint Surg Am, 84A(5): 793-799

Nimni ME. 1997, Polypeptide growth factors: targeted delivery systems. Biomaterials, 18(18): 1201-1225

O'Brien FJ. 2011, Biomaterials and scaffolds for tissue engineering. Materials Today, 14(3): 88-95

Olde Damink LH, Dijkstra PJ, van Luyn MJ, van Wachem PB, Nieuwenhuis P, Feijen J. 1996, Cross-linking of dermal sheep collagen using a watersoluble carbodiimide. Biomaterials, 17(8): 765-773.

Phinney DG, Kopen G, Righter W, Webster S, Tremain N, Prockop DJ. 1999, Donor variation in the growth properties and osteogenic potential of human marrow stromal cells. J Cell Biochem, 75(3): 424-436.

Pilgaard L, Lund P, Duroux M, Fink T, Ulrich-Vinther M, Soballe K, Zachar V. 2009, Effect of oxygen concentration, culture format and donor variability on in vitro chondrogenesis of human adipose tissue-derived stem cells. Regen Med, 4(4): 539-548 
Roberts AB, Flanders KC, Heine UI, Jakowlew S, Kondaiah P, Kim SJ, Sporn MB. 1990, Transforming growth factor- $\beta$ : multifunctional regulator of differentiation and development. Philos Trans $R$ Soc Lond B Biol Sci, 327(1239): 145-154

Rowland CR, Lennon DP, Caplan AI, Guilak F. 2013, The effects of crosslinking of scaffolds engineered from cartilage ECM on the chondrogenic differentiation of MSCs. Biomaterials, 34(23): 5802-5812

Shintani N, Hunziker EB. 2011, Differential effects of dexamethasone on the chondrogenesis of mesenchymal stromal cells: Influence of microenvironment, tissue origin and growth factor. European Cells and Materials, 22: 302-320

Solorio LD, Dhami CD, Dang PN, Vieregge EL, Alsberg E. 2012, Spatiotemporal regulation of chondrogenic differentiation with controlled delivery of transforming growth factor- $\beta 1$ from gelatin microspheres in mesenchymal stem cell aggregates. Stem Cells Tran Med, 1(8), 632-639

Solorio LD, Vieregge EL, Dhami CD, Dang PN, Alsberg E. 2012, Engineered cartilage via self-assembled hMSC sheets with incorporated biodegradable gelatin microspheres releasing transforming growth factorß1. Journal of Controlled Release, 158(2): 224-232

Sutherland AJ, Converse GL, Hopkins RA, Detamore MS. 2015, The bioactivity of cartilage extracellular matrix in articular cartilage regeneration. Advanced Healthcare Materials, 4(1): 29-39 
Tang QO, Shakib K, Heliotis M, Tsiridis E, Mantalaris A, Ripamont U, Tsiridis E. 2009, TGF- $\beta 3$ : A potential biological therapy for enhancing chondrogenesis. Expert Opin Biol Ther, 9(6): 689-701

van Beuningen HM, Glansbeek HL, van der Kraan PM, van den Berg WB. 1998, Differential effects of local application of BMP-2 or TGF- $\beta 1$ on both articular cartilage composition and osteophyte formation. Osteoarthritis Cartilage, 6(5): 306-317

van den Berg WB, van Osch GJ, van der Kraan PM, van Beuningen HM. 1993, Cartilage destruction and osteophytes in instability-induced murine osteoarthritis: role of TGF- $\beta$ in osteophyte formation? Agents Actions, 40(3-4): $215-219$

van der Kraan PM, van den Berg WB. 2007, Osteophytes: relevance and biology. Osteoarthritis Cartilage, 15(3): 237-244

Vinatier C, Mrugala D, Jorgensen C, Guicheux J, Noel D. 2009, Cartilage engineering: a crucial combination of cells, biomaterials and biofactors. Trends Biotechnol, 27(5): 307-314

Vonwil D, Wendt D, Ströbel S, Wallny HJ, Gygax D, Heberer M, Martin I. 2008, Assessment of the stability of TGF- $\beta 3$ bioactivity for potential bioreactor applications. Biochemical Engineering Journal, 39(3): 586-589

Wilson CG, Nishimuta JF, Levenston ME. 2009, Chondrocytes and meniscal fibrochondrocytes differentially process aggrecan during de novo extracellular matrix assembly. Tissue Eng Part A, 15(7): 1513-1522 
Worster AA, Nixon AJ, Brower-Toland BD, Williams J. 2000, Effect of transforming growth factor- $\beta 1$ on chondrogenic differentiation of cultured equine mesenchymal stem cells. Am J Vet Res, 61(9):1003-1010

Young S, Wong M, Tabata Y, Mikos AG. 2005, Gelatin as a delivery vehicle for the controlled release of bioactive molecules. J Control Release, 109(13): $256-274$

\section{Figure Captions}

Figure 1 - Scanning electron micrographs of the porous ECM-derived scaffold in low $(A)$ and high $(B)$ magnification.

Figure 2 - Total cumulative TGF- $\beta 3$ content (ELISA) of the culture media for day 3, 7 and 12 of culture for low (A), medium (B) and high (C) growth factor loaded groups $(n=3)$. Release profile into the media for the first 12 days of culture for low (D), medium (E) and high (F) TGF- $\beta 3$ loaded groups $(n=3)$.

Figure 3 - Macroscopic images of ECM-derived scaffolds seeded with infrapatellar fat pad-derived stem cells after 4 weeks in culture for no TGF- $\beta 3$ (A), low (E), medium (I), high (M) and direct media TGF- $\beta 3$ supplementation (Q). Alcian blue, picro-sirius red and type II collagen staining for no TGF- $\beta 3$ (BD), low $(\mathrm{F}-\mathrm{H})$, medium (J-L), high (N-P) and direct media TGF- $\beta 3$ supplementation $(\mathrm{R}-\mathrm{T})$. Scale bar: $50 \mu \mathrm{m}$.

Figure 4 - (A) sGAG and (B) collagen accumulation within ECM-derived constructs seeded with infrapatellar fat pad-derived stem cells after 4 weeks of culture for media, low, medium and high TGF- $\beta 3$ supplementation $(n=5$, $\left.{ }^{*} p<0.05\right)$. Dashed line represents day 0 values for acellular scaffolds. 
Figure 5 - Alcian blue and picro-sirius red staining for low, medium, high and media TGF- $\beta 3$ supplementation for six different donors (A-F), healthy and diseased (osteoarthritic - OA). All micrographs are for 4 weeks in culture with human infrapatellar fat pad-derived stem cells. Scale bar: $50 \mu \mathrm{m}$.

Figure 6 - sGAG accumulation within ECM-derived constructs after 4 weeks culture period with infrapatellar fat pad-derived stem cells for media, low, medium and high TGF- $\beta 3$ supplementation for six different donors (A-F) ( $n=5$, $\left.{ }^{*} p<0.05\right)$. Dashed line represents day 0 value for acellular scaffolds.

\section{Fig 1}
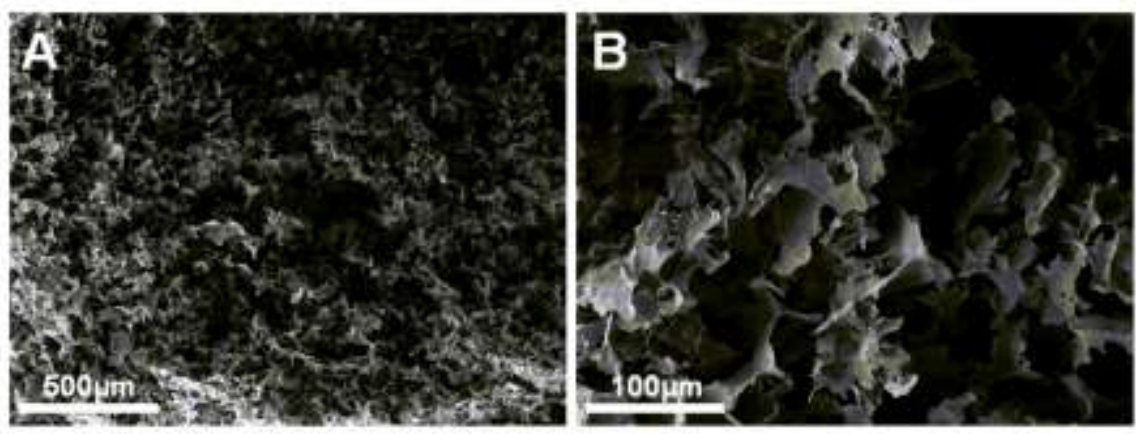
Fig 2

A
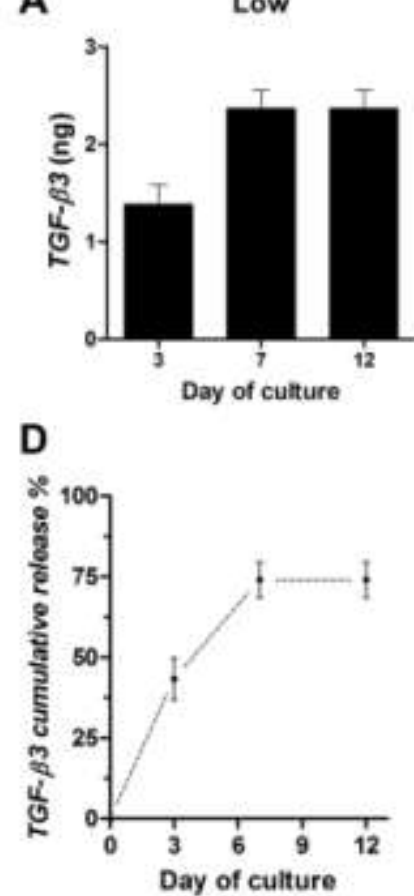

B

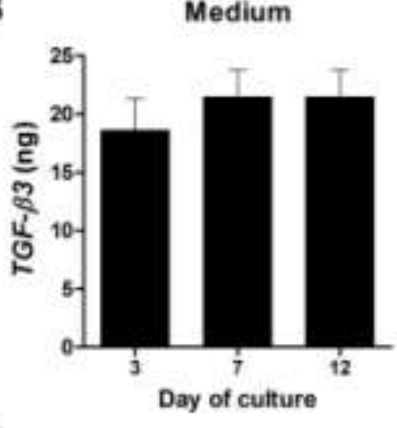

E

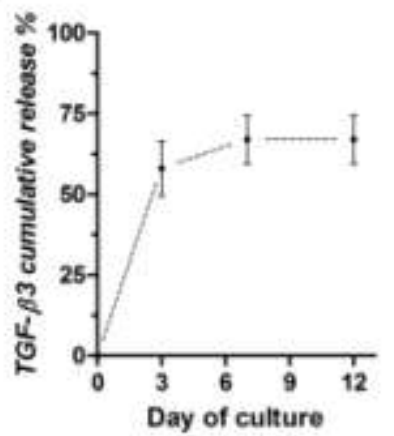

C

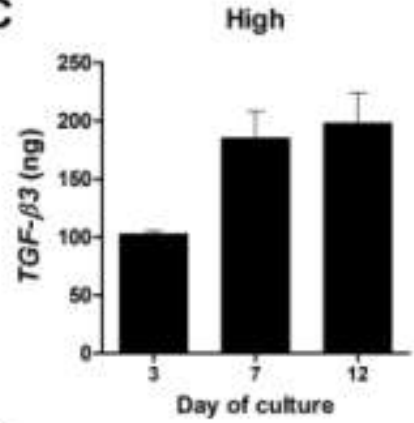

F

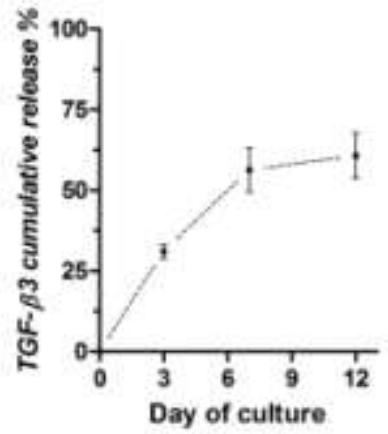


Fig 3

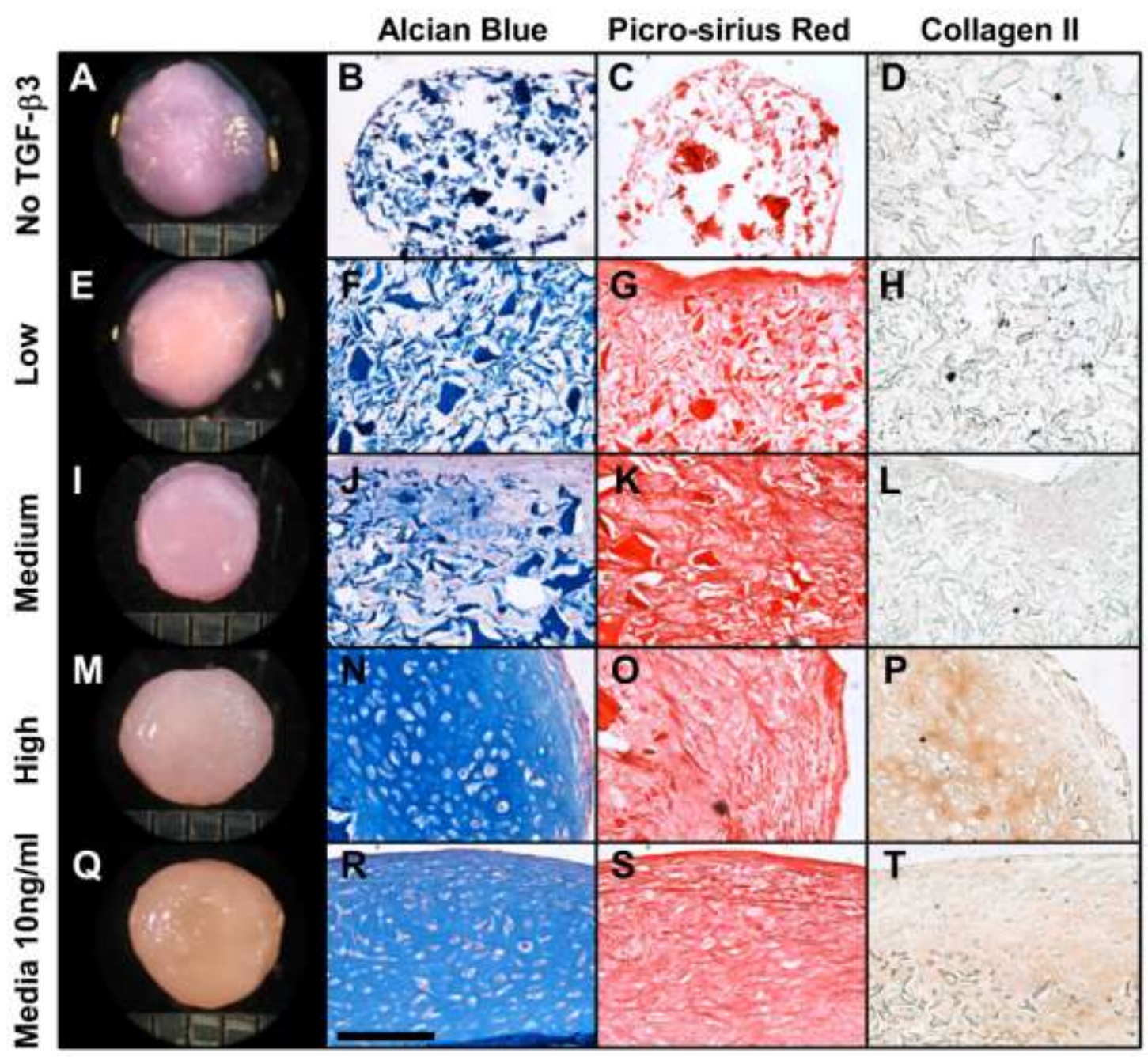

Fig 4
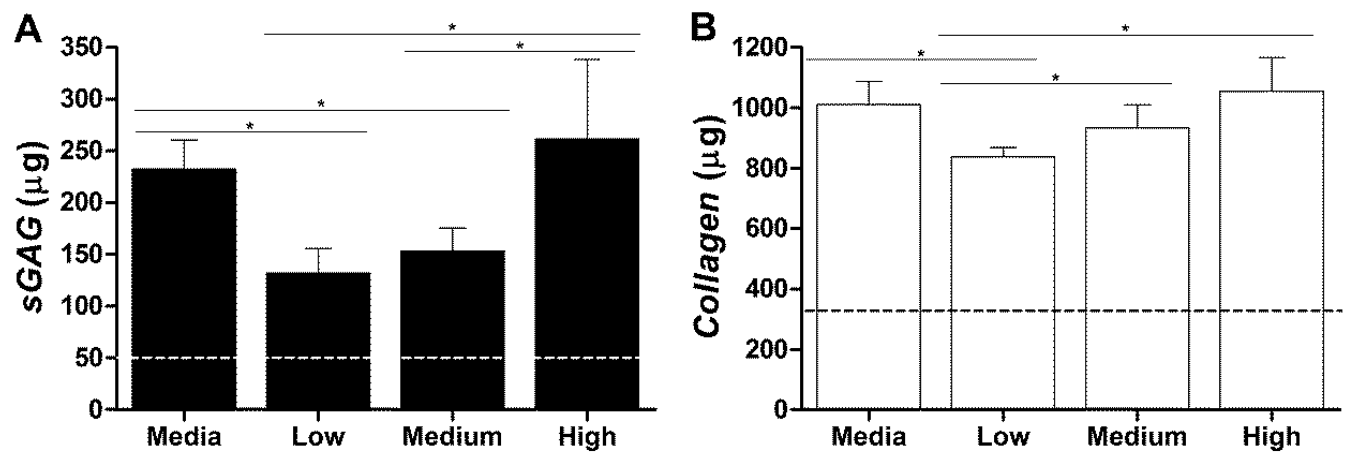
Fig 5

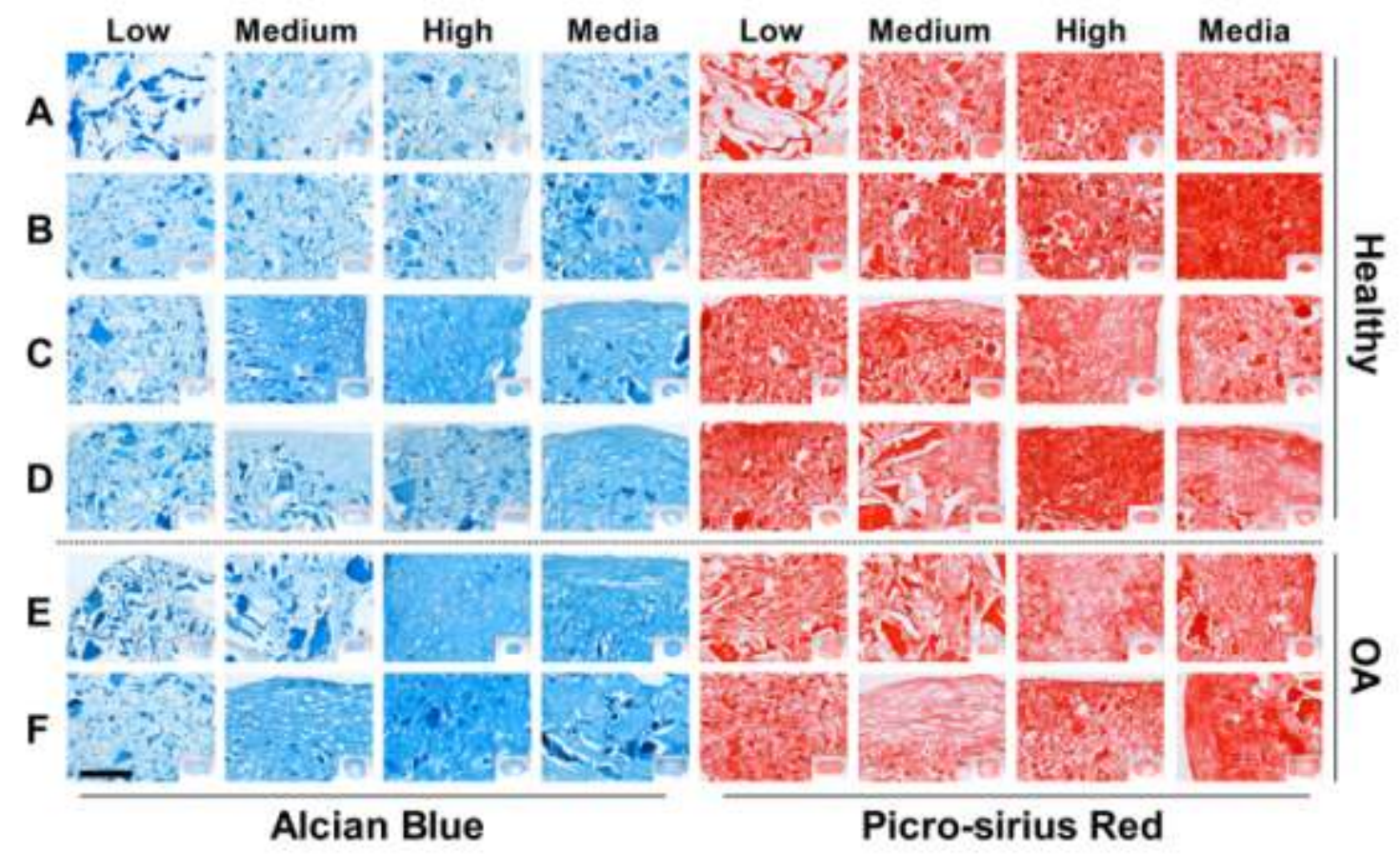

Fig 6

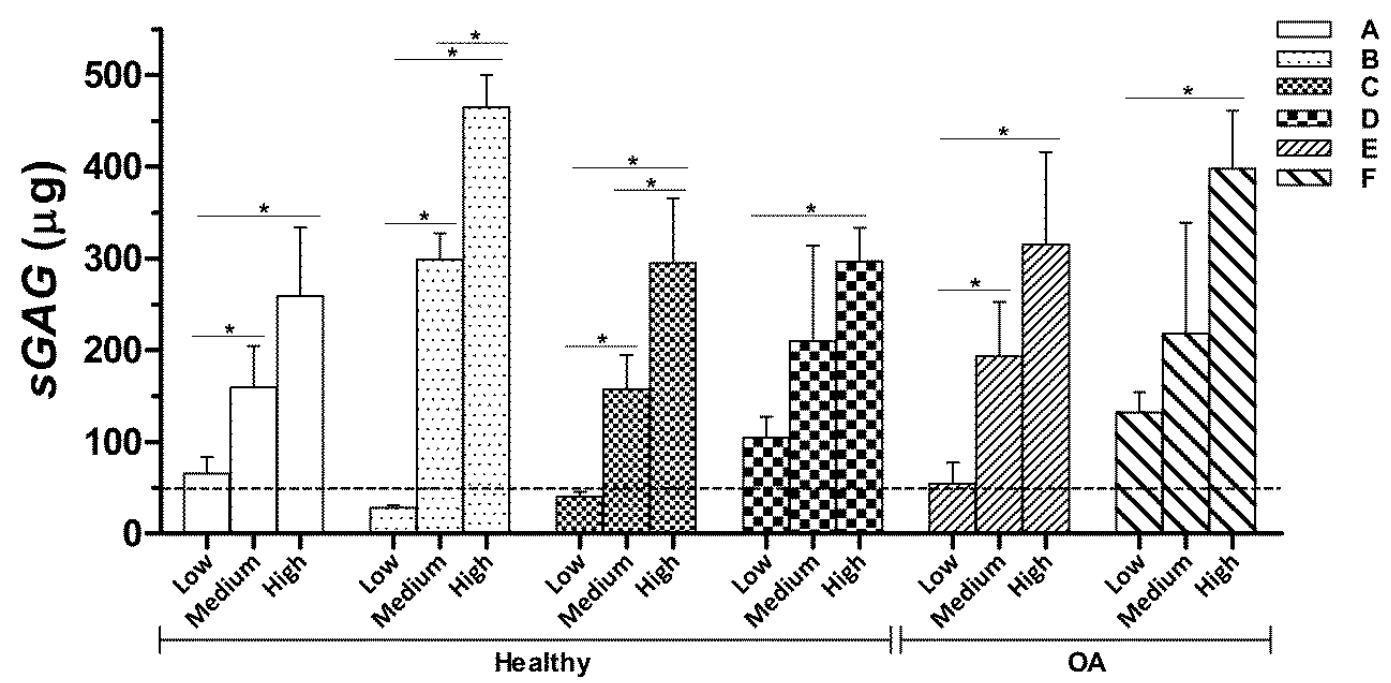

